\title{
Cohort retrospective study: the neutrophil to lymphocyte ratio as an independent predictor of outcomes at the presentation of the multi-trauma patient
}

\author{
Soulaiman Elias Soulaiman ${ }^{1 *}$ D, Dalal Dopa², Al-Batool T. Raad², Walaa Hasan², Niyazi Ibrahim³, Al-Ykzan Hasan³,
} Hussam Aldin Sulaiman ${ }^{4}$ and Moufid Darwich ${ }^{4}$

\begin{abstract}
Background: Although the association of neutrophil to lymphocyte ratio (NLR) with mortality in trauma patients has recently been shown, there is a paucity of research on the association with other outcomes. Recent studies suggest that the NLR has a predictive value of mortality in trauma patients during various times of admission. This study aimed to determine the prognostic impact of NLR at the presentation in critically ill trauma patients.

Methods: A retrospective cohort study of adult trauma patients between July 2017 and November 2017 in Tishreen Hospital. All patients who had arrived at the emergency department with multi-trauma injury within the age category (14-80 years) were included in this analysis. The prophetical capability of NLR on mortality was assessed by the receiver operative characteristics (ROC) curve. To identify the impact of the NLR on survival, a separate log-rank test was used. Multivariable Cox proportional hazard modeling was used to identify independent predictors of mortality.

Results: Throughout the time of the study, 566 patients met the inclusion criteria. Of these, $98.8 \%$ were male, $75.8 \%$ sustained penetrating trauma, and median age [IQR25-IQR75] was 26 [23-32]. Ninety-seven patients (17.1\%) had major trauma, with an Injury Severity Score (ISS) $\geq 15$. Using the ROC curve analyses hospitalization day 1, optimal NLR cutoff values of 4.00 were calculated by maximizing the Youden index. Kaplan-Meier curves revealed an NLR greater than or equal to these cutoff values as a marker for increased in-hospital mortality ( $p=0.020$, log-rank test). The Cox regression model demonstrated significant collinearity among the predictive variables (all VIF results $<2$ ). Only ISS $>15$ has a significant statistical relation with elevated NLR on day $1(p=0.010)$.
\end{abstract}

Conclusions: Elevated NLR on day 1 has high predictive power for overall survival during the first 30 days after trauma, but it was not independent of other factors.

Keywords: Neutrophil to lymphocyte ratio, Trauma, Intensive care, Mortality

\section{Background} Injuries

According to the World Health Organization, injuries are still a major cause of severe disability and death. Traumarelated injuries result in the death of more than five million people worldwide annually accounting for approximately $9 \%$ of global mortality [1]. The care of severely injured

\footnotetext{
* Correspondence: soulaimanelias@gmail.com

${ }^{1}$ Department of Hematology and Bone Marrow Transplantation, Tishreen Hospital, Damascus, Syria

Full list of author information is available at the end of the article
}

patients remains a challenge, which makes trauma death studies have importance as these proceed with a medical audit and a measure for quality of care provided to trauma patients within the prehospital and in-hospital setting.

The classical trimodal distribution of trauma deaths describes three peaks of deaths following trauma: immediate, early, and late deaths [2]. The predominant cause of death after trauma continues to be central nervous system (CNS) injury, followed by exsanguination, while sepsis and multi-organ failure (MOF) continue to be predominant causes of late death [3]. 


\section{Prognosis and risk factors}

One of the important factors that increase mortality in post-traumatic patients is pathological pro- and antiinflammatory responses occurring in the first hours after extensive trauma with massive infection, which is still difficult to control and to discriminate from a physiological immune response [4]. In recent years, there has been a particular focus on the balance of this pro-inflammatory response and the anti-inflammatory response as systemic predictors of outcomes in critically ill patients. Patients often develop numerous abnormalities in their host defense systems in response to severe injury [5]. Problems arise when this unbalanced inflammatory response escalates and releases an excess of pro-inflammatory mediators such as IL-1, IL-6, IL-8, and TNF $\alpha$, culminating in systemic inflammatory response syndrome (SIRS) [6]. Progression of this uncontrolled cytokine cascade and hyperinflammation augments the injury burden, resulting in deleterious and often life-threatening events such as sepsis and multiple organ dysfunction syndrome (MODS).

\section{The neutrophil to lymphocyte ratio}

In contrast to the widespread activation of neutrophils, post-injury is the fall in total lymphocyte levels which occurs in response to multiple trauma. The prognostic value of neutrophil to lymphocyte ratio (NLR) had estimated in non-traumatic disorders where studies showed NLR is effective in the diagnosis of familial Mediterranean fever [7]; it has also been studied for acute appendicitis and has been found to have a higher sensitivity than leukocytosis [8]. For this reason, the NLR can potentially be used as an early indicator of inflammatory homeostasis derailment in patients with tissue injury.

\section{Aim of the study}

We conducted this study in trying to assess the NLR in multi-trauma patients at presentation as a useful predictor of worse outcomes, and then it can be used to help build a prognostic model.

\section{Methods}

\section{Study design}

This study is a retrospective cohort study of adult trauma patients between July 2017 and November 2017 in Tishreen Hospital. All patients who had arrived at the emergency department with multi-trauma injury within the age category (14-80 years) were included in this analysis.

All patients coming directly from the site of injury or referred from another hospital to the emergency department were subjected to the same criteria after excluding those who spent more than $24 \mathrm{~h}$ to arrive in our hospital. During war conditions, it was difficult to obtain accurate data about the initial approach of the injured due to the enormous workload and the difficulty of mobility where the priority was to save lives.

Patients were excluded if they were below 14 years of age because there is a wide variation in the number of lymphocytes within this age group that may affect the estimation for the prognostic value of NLR. We also excluded patients who had expired in the emergency department or the operating room because it was difficult to get all variables that we need for our study given the change in the value of NLR during the hospitalization period and its relationship with other variables.

For the purpose of the study, two trauma team experts, one collected laboratory data and other followed clinical and surgical issues, performed extensive chart and computer checks for data completeness, accuracy, and consistency.

Demographic and clinical data elements from the respective medical files collected by trained chart abstractor then computerized into database excel file included age, gender, Injury Severity Score (ISS), mechanism of injury (MOI), initial vital signs upon presentation to the emergency department (ED), hospital length of stay (LOS), intensive care unit LOS (ICU LOS), blood cell count with differential, and in-hospital mortality.

\section{Primary outcome variable}

The primary variable is the NLR at admission (day 1), which is defined as the ratio of absolute neutrophil to absolute lymphocytes taken from the data of the laboratory department at the admission in the emergency department.

We considered cutoff for NLR equal to 4 to study patients in two groups to determine the prognostic value of this variable on the overall survival and mortality.

\section{Secondary outcome variables}

The secondary variables are age; gender; ISS, calculated by a scientific calculator by entering the required data after obtaining it from the patient files; MOI, with two types of injuries identified as penetrating and blunt; initial vital signs upon presentation to the ED; LOS; and ICU LOS, aiming to build epidemiological study and screen for the risk factors.

\section{Statistical analysis}

The data were analyzed by the IBM SPSS v.22 program for the normal distribution of continuous variables by using histograms and the Shapiro-Wilk test. Continuous variables were reported as medians and interquartile ranges (IQR). Categorical variables were reported as frequencies and percentages. To examine the differences between NLR groups, categorical covariates were analyzed using Fisher's exact test, while the continuous covariates were analyzed based on Mann-Whitney $U$ test. The predictive capacity of the NLR on mortality was assessed using receiver operating characteristics (ROC) 
curve analysis. The NLR analysis was performed for hospital day 1 . These periods were selected to adjust for the clinical probability of early and late complications. Optimum cutoff values were determined on the ROC curve with the maximum Youden index [sensitivity-(1specificity)]. Baseline characteristics and outcome variables of the high and the low NLR groups were compared in a univariate analysis. The in-hospital mortality during the study period was assessed using Kaplan-Meier curves, and the log-rank test was used for between-group comparisons for the NLR cutoff values on hospital day 1 .

\section{Results}

During the study time frame, baseline characteristics of patients are shown in Table 1: a total of 566 trauma patients aged 14 and older were admitted to an emergency department in Tishreen Hospital, and patients arrived in the hospital by two methods: directly from the place of accident or transferring from another hospital. Five hundred and sixty (98.8\%) were male. Median age [IQR25IQR75] was 26 [23-32], and $42.2 \%$ of patients were older than 26 years. Penetrating injury was seen in 429 (75.8\%) of the patients, and 97 (17.1\%) patients had major trauma, with an ISS $\geq 15$. Only six patients $(1.1 \%)$ had damage control surgery (DCS).

All patients (566) had hospitalization, $12.9 \%$ of them $(n=73)$ were hospitalized for more than 7 days, 65 patients needed ICU, and $4.1 \%(n=23)$ of these patients stayed in ICU for more than 7 days. At admission to the emergency department, only $17.1 \%(n=97)$ had systolic blood pressure less or more than $90 \mathrm{~mm}$.

For the NLR on day 1, the AUC was 0.633 (95\% CI $0.542-0.725, p=0.080$ ). The ROC curve analysis for hospital day 1 revealed optimal cutoff values of 4 , corresponding to a sensitivity of $70.3 \%$ and specificity of $56.4 \%$ (Fig. 1).

When baseline characteristics were compared (Table 2), for the high and low NLR groups on hospital day 1, in the high NLR group, age $\geq 26$ years $(p=0.758)$, systolic blood

Table 1 Baseline characteristics of patients admitted to Tishreen Hospital during the study period

\begin{tabular}{ll}
\hline & $N=566$ \\
\hline Age $>26$ & $239(42.2 \%)$ \\
Male gender & $506(98.8 \%)$ \\
ISS $>15$ & $97(17.1 \%)$ \\
Penetrate injury & $429(75.8 \%)$ \\
LOS $>7$ days & $73(12.9 \%)$ \\
ICU admission & $65(11.5 \%)$ \\
ICU LOS $>7$ days & $23(4.1 \%)$ \\
SBP $<90$ & $97(17.1 \%)$ \\
DCS & $6(1.1 \%)$ \\
\hline
\end{tabular}

ISS Injury Severity Score, LOS length of stay in hospital, ICU intensive care unit, $S B P$ systolic blood pressure, DCS damage control surgery

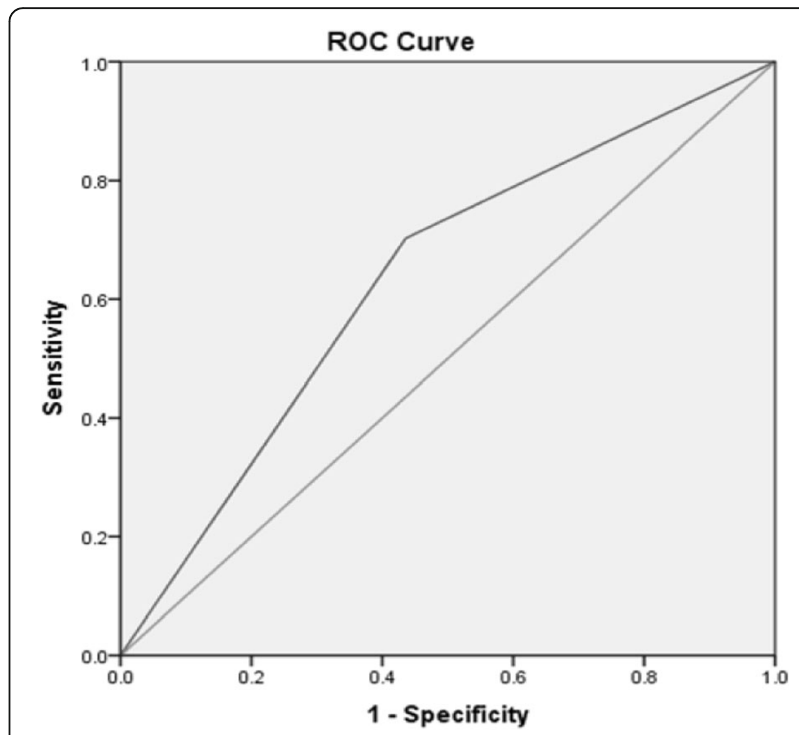

Fig. 1 The ROC curve analysis of neutrophil to lymphocyte ratio (NLR) at time admission (day 1) revealed optimal cutoff values of 4 , corresponding to a sensitivity of $70.3 \%$ and a specificity of $56.4 \%$

pressure $(\mathrm{SBP}) \leq 90 \mathrm{mmHg}(p=0.662)$, penetrating injury $(p=1.000)$, lengthy stay in hospital LOS $>7$ days $(p=$ $0.228)$, length stay in ICU $>7$ days $(p=0.229)$, all have no statistical significance between high and low NLR groups. Only ISS $>15(p=0.010)$ has statistical significance (Fig. 2).

When the Kaplan-Meier curves (Fig. 3) of the overall survival were compared, a statistically significant difference was observed between the patients above and below the NLR cutoff values for day 1 hospitalization $(p=0.020)$.

The regression models demonstrated significant collinearity among the predictive variables (all VIF results $<2$ ). Significant interactions were identified between the NLR, time, and the other predictive variables (including age $\geq$ 26 , $\mathrm{SBP} \leq 90$, MOI penetrate vs blunt, ISS > 15).

\section{Discussion}

Outcome prediction in the critically ill trauma patients using readily available, easily obtainable, simple, and repeatable clinical data points is an important research goal that to date has not been adequately achieved. NLR is a simple, stand-alone method for evaluating systemic inflammation and outcomes in trauma patients.

During war conditions, the number of injuries coming to the emergency department is increasing, and the types of injury vary beyond the ability of the medical staff to deal decisively and effectively with all cases. In such a situation, mostly, the first step is categorizing and managing the injuries according to their risk. To achieve this step, we need an easy, fast, and credible prognostic scale. A review of the literature found several studies on prognostic factors for trauma patients, which suggested that the predictive capacity of the well-known scoring 
Table 2 The relation between baseline characteristics and neutrophil to lymphocyte ratio (NLR) at admission (day 1)

\begin{tabular}{llll}
\hline & NLR $>4$ on day $1(n, 165)$ & NLR $<4$ on day $1(n, 184)$ & $p$ value \\
\hline Age $>26$ years & $74(41.3 \%)$ & $105(58.7 \%)$ & 0.758 \\
ISS $>15$ & $45(25.1 \%)$ & $25(12.0 \%)$ & $0.010^{*}$ \\
Penetrate injury & $134(74.9 \%)$ & $156(74.6 \%)$ & 1.000 \\
LOS $>7$ days & $28(15.6 \%)$ & $23(11 \%)$ & 0.228 \\
ICU LOS $>7$ days & $12(7.5 \%)$ & $5(2.7 \%)$ & 0.229 \\
SBP $<90$ & $166(94.9 \%)$ & $201(96.2 \%)$ & 0.662 \\
\hline
\end{tabular}

ISS Injury Severity Score, LOS length of stay in hospital, ICU intensive care unit, SBP systolic blood pressure

*statistical significance

systems such as the admission ISS are not as good as the several well-validated physiologic-based scoring models such as the Denver, Sequential Organ Failure Assessment (SOFA), Acute Physiology and Chronic Health Evaluation II (APACHE II), Revised Trauma Score, and Trauma and Injury Severity Score (TRISS) [9]. However, the predictive capacities of these physiologic-based models are complicated and too unwieldy for daily use and require a long time to apply.

Few studies have evaluated the association between NLR and outcomes in multi-trauma patients, demonstrating worse outcomes with increasing NLR. A recent study had found that a trajectory of increasing NLR over the first $48 \mathrm{~h}$ of admission is associated with the development of organ failure among male trauma patients [10]. Another study realizes that NLR is strongly associated with early mortality in trauma patients with severe hemorrhage managed with multi-transfusion protocol (MTP) [11]. According to Dilektasli et al. who demonstrated the prognostic role of NLR at days 2 and 5 in predicting hospital deaths in trauma patients, in contrast, the NLR in the first $24 \mathrm{~h}$ was not useful for

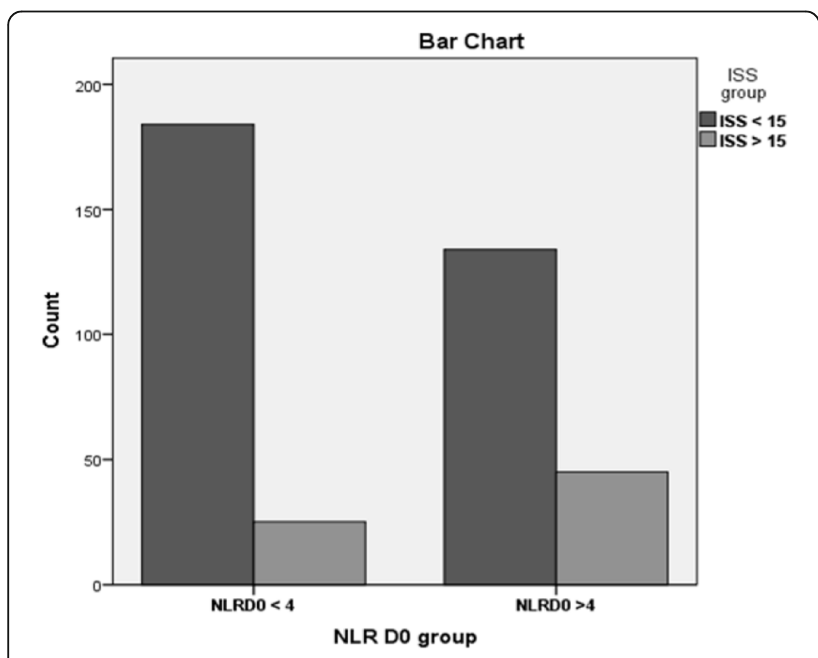

Fig. 2 The relation between Injury Severity Score (ISS) and neutrophil to lymphocyte ratio (NLR) at admission (day 1); ISS $>15$ has statistical significance $(p=0.010)$ predicting outcomes when compared with the next days in the surgical intensive care unit [12]; in this study, hospital days 2 and 5 revealed optimal cutoff values of 8.19 and 7.92, corresponding to a sensitivity and specificity of $70.8 \%$ and $61.9 \%$ and $75.6 \%$ and $66.9 \%$, respectively. According to Forget et al., BMC Res Notes, the normal NLR values in an adult, non-geriatric, population in good health are between 0.78 and 3.53 [13]. So, our study for hospital day 1 revealed optimal cutoff values of 4 , corresponding to a sensitivity of $70.3 \%$ and specificity of $56.4 \%$.

Our study showed an important prognostic value for NLR during the first $24 \mathrm{~h}$ of receiving patients in the emergency department to predict survival within 30 days following the admission.

\section{Conclusion}

Elevated NLR during the first $24 \mathrm{~h}$ of admission (day 1 ) has high predictive power for overall survival during the first 30 days after trauma, but it was not independent of other factors.

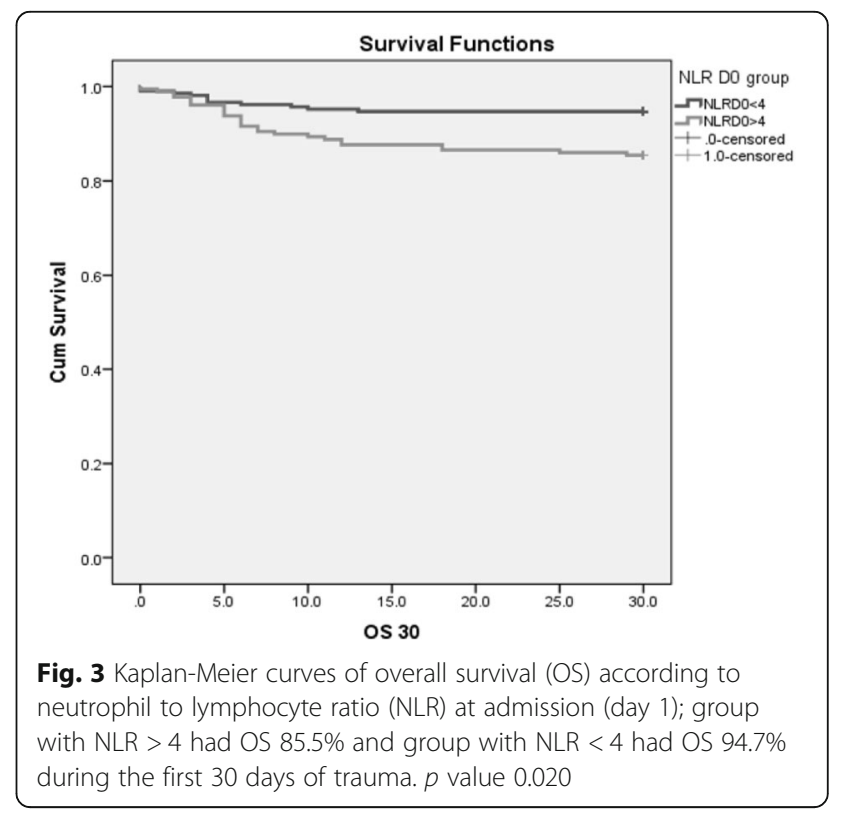


We found a closed relation between a high level of ISS and elevated NLR on day 1 which guided for more studies in trying to build a new prognostic model for multitrauma patients which included ISS and NLR at admission. To achieve that, a prospective external validation is warranted in a large trauma population.

This study is a retrospective database study; the nature of this study may lead to error due to confounding and bias for patient and treatment selections.

\section{Acknowledgements}

We would like to extend our gratitude to Dr. Ammar Suleiman, Head of Medical Services Institution, for his support and facilitation and to the medical staff working in the emergency department for their assistance provided in the extraction of patient data.

\section{Authors' contributions}

SES conceived and designed this study. ABTA, WH, and NI collected the data. SES and $\mathrm{NI}$ prepared the figures and conducted the data analyses. SES, MD, DD, and HAS drafted the manuscript. All authors interpreted the data and critically revised the manuscript. All authors have read and approved the final version of the manuscript.

\section{Funding}

The authors received no external funding.

\section{Availability of data and materials}

The datasets used during the current study are available from the corresponding author on a reasonable request.

\section{Ethics approval and consent to participate}

The Regional Ethics Committee (REC) was informed about the study and deemed the study a clinical quality study not needing formal REC approval. All institutional bodies waived the need for patient consent.

\section{Consent for publication}

Not applicable.

\section{Competing interests}

The authors declare that they have no competing interests.

\section{Author details}

'Department of Hematology and Bone Marrow Transplantation, Tishreen Hospital, Damascus, Syria. ${ }^{2}$ Department of Laboratory, Tishreen Hospital, Damascus, Syria. ${ }^{3}$ Department of Surgery, Tishreen Hospital, Damascus, Syria.

${ }^{4}$ Department of Orthopedic Surgery, Tishreen Hospital, Damascus, Syria.

Received: 9 September 2019 Accepted: 21 January 2020

Published online: 04 February 2020

\section{References}

1. WHO | Injuries and violence: the facts 2014 [Internet]. WHO. [cited 2019 Nov 28]. Available from: https://www.who.int/violence_injury_prevention/media/ news/2015/Injury_violence_facts_2014/en/

2. Trunkey DD. Trauma. Accidental and intentional injuries account for more years of life lost in the U.S. than cancer and heart disease. Among the prescribed remedies are improved preventive efforts, speedier surgery, and further research. Sci Am. 1983 Aug;249(2):28-35.

3. Dewar DC, White A, Attia J, Tarrant SM, King KL, Balogh ZJ. Comparison of postinjury multiple-organ failure scoring systems: Denver versus sequential organ failure assessment. J Trauma Acute Care Surg. 2014;77(4):624-9.

4. Current views on the mechanisms of immune responses to trauma and infection [Internet]. [cited 2019 Nov 28]. Available from: https://www.ncbi. nlm.nih.gov/pmc/articles/PMC4637396/

5. Early predictors of postinjury multiple organ failure| JAMA Surgery | JAMA Network [Internet]. [cited 2019 Apr 29]. Available from: https://jamanetwork. com/journals/jamasurgery/article-abstract/595844

6. Lord JM, Midwinter MJ, Chen Y-F, Belli A, Brohi K, Kovacs EJ, et al. The systemic immune response to trauma: an overview of pathophysiology and treatment. Lancet. 2014;384(9952):1455-65.
7. Ahsen A, Ulu MS, Yuksel S, Demir K, Uysal M, Erdogan M, et al. As a new inflammatory marker for familial Mediterranean fever: neutrophil-tolymphocyte ratio. Inflammation. 2013;36(6):1357-62.

8. Yazici M, Ozkisacik S, Oztan MO, Gürsoy H. Neutrophil/lymphocyte ratio in the diagnosis of childhood appendicitis. Turk J Pediatr. 2010;52(4):400-3.

9. Hwang SY, Lee JH, Lee YH, Hong CK, Sung AJ, Choi YC. Comparison of the sequential organ failure assessment, acute physiology and chronic health evaluation II scoring system, and trauma and injury severity score method for predicting the outcomes of intensive care unit trauma patients. Am J Emerg Med. 2012;30(5):749-53.

10. Younan D, Richman J, Zaky A, Pittet J-F. An increasing neutrophil-tolymphocyte ratio trajectory predicts organ failure in critically-ill male trauma patients. An exploratory study. Healthcare. 2019;7(1):42

11. Duchesne JC, Tatum D, Jones G, Davis B, Robledo R, DeMoya M, et al. Multiinstitutional analysis of neutrophil-to-lymphocyte ratio (NLR) in patients with severe hemorrhage: a new mortality predictor value. J Trauma Acute Care Surg. 2017;83(5):888-93.

12. Dilektasli E, Inaba K, Haltmeier T, Wong MD, Clark D, Benjamin ER, et al. The prognostic value of neutrophil-to-lymphocyte ratio on mortality in critically ill trauma patients. J Trauma Acute Care Surg. 2016;81(5):882-8.

13. Forget $P$, Khalifa C, Defour J-P, Latinne D, Van Pel M-C, De Kock M. What is the normal value of the neutrophil-to-lymphocyte ratio? BMC Res Notes. 2017;10(1):12

\section{Publisher's Note}

Springer Nature remains neutral with regard to jurisdictional claims in published maps and institutional affiliations.
Ready to submit your research? Choose BMC and benefit from:

- fast, convenient online submission

- thorough peer review by experienced researchers in your field

- rapid publication on acceptance

- support for research data, including large and complex data types

- gold Open Access which fosters wider collaboration and increased citations

- maximum visibility for your research: over $100 \mathrm{M}$ website views per year

At $\mathrm{BMC}$, research is always in progress.

Learn more biomedcentral.com/submission 